\title{
Une traversée en théorie intertextuelle : « Les doigts extravagants » d'Andrée Maillet
}

\author{
Rebecca Josephy \\ Université de Western Ontario \\ et Université Paris Ouest Nanterre
}

Imaginez que vous vous promeniez dans les rues de New York. Un inconnu vous donne un manteau dans la poche duquel vous trouvez « cinq doigts d'une main gauche d'homme coupés audessus des phalanges et reliés entre eux par un lacet » (Maillet, 1987, p. 150). Vous faites tout pour vous débarrasser des doigts, mais ils s'animent, grimpent aux murs de votre appartement, vous suivent, vous tourmentent. Et puis, ils commencent à griffonner un message sur votre table de cuisine : «Garde-nous. Sans âme nous nous désagrégerons. Prêtenous la tienne. [...] Nous ne demandons rien d'autre que l'appui 
de ta pensée. Prête-nous ta vie, nous ferons ta fortune. » (p. 153) Vous acquiescez et, le pacte scellé, les doigts se mettent à écrire une œuvre qui vous apportera un énorme succès financier et critique. Existez-vous toujours? Ainsi se résume la nouvelle fantastique « Les doigts extravagants » d'Andrée Maillet.

L'idée d'être poursuivi, voire hanté par l'élan créateur d'un autre soulève la problématique de la filiation littéraire. Comment écrire après ceux que l'on a lus? C'est une question qui est étroitement liée à l'intertextualité, car, comme le propose Harold Bloom dans The Anxiety of Influence, l'absence ou la présence dans le texte d'influence est révélatrice d'un positionnement par rapport aux précurseurs ; tout texte écrit suit un autre texte, tout écrivain s'éloigne ou se rapproche de ses prédécesseurs, les meilleures œuvres étant, selon lui, le fruit de lectures décalées. Néanmoins, le lien entre influence et intertextualité n'a pas toujours été évident. $\mathrm{Au}$ contraire, comme le soulignent Clayton et Rothstein,

[o]ver the last two decades, the concepts of influence and intertextuality have been sites of generational conflict: to many people, influence has smacked of elitism, the old boy networks of Major Authors and their sleek entourages; others have suspected that the intertextual web flowed from the needles of Mme. Defarge. More routinely, the new and voguish "intertextuality" has served as a generational marker for younger critics...1 (p. 3 )

\footnotetext{
1 Je traduis. Au cours des deux dernières décennies, les concepts d'influence et d'intertextualité ont été le théâtre de conflits intergénérationnels : pour un certain nombre, la notion d'influence avait la couleur de l'élitisme, des réseaux traditionnels des auteurs canoniques et des gardiens du temple; d'autres suspectaient que la maille de l'intertextualité venait de la pelote de Madame Defarge. Plus couramment, le concept en vogue d'intertextualité a servi de marqueur générationnel pour de jeunes critiques.
} 
Ce clivage était donc dû, en large partie, à la nécessité de fonder le concept d'intertextualité sur une rupture avec les outils conceptuels du passé. En ce sens, au contraire d'une opposition un tant soit peu artificielle, nous voyons dans la nouvelle de Maillet qu'il est possible, du moins en littérature, de naviguer naturellement entre les eaux de l'influence et celles de l'intertextualité. Des fragments de phrases, des références, des mots venant d'autres œuvres fantastiques s'imposent dans le récit comme la manifestation physique de l'anxiété ressentie par l'écrivaine face aux «influences ». C'est une nouvelle offrant donc des ouvertures sur d'autres œuvres - une référence en entraînant une autre - , en trop grand nombre, sans doute, pour en faire un décompte exhaustif. Cependant, tout aussi important que le nombre étonnant de références intertextuelles est le fait que l'origine des mots apparaisse explicitement dans le récit, et ce, avant la majorité des théories dans le domaine. Depuis l'invention du terme « intertextualité » par Kristeva en 1966 (trois ans après la publication de la nouvelle de Maillet ${ }^{2}$ ), le concept se complique, évolue sans cesse et se fragmente en raison du nombre de définitions qui s'y rattachent. La question qui se pose est donc la suivante: à la lumière de ces théories divergentes, que peut apporter au débat et à l'histoire de l'intertextualité la nouvelle de Maillet, profondément perturbée par l’origine des mots?

\section{Quand les polyphonies s'entendent}

Le mot «intertextualité » apparaît d'abord dans un article de Tel Quel, «Le mot, le dialogue, le roman» (1966), et se

2 Originellement cette nouvelle paraît dans le recueil Le lendemain n'est pas sans amour, publié en 1963. 
développe au fur et à mesure des recherches subséquentes de Kristeva : "Le texte clos », également dans Tel Quel (1967), et Séméiotikè, Recherches pour une sémanalyse (1969). Au travers de ces travaux matriciels, une définition se cristallise : «le mot (le texte) est un croisement de mots (de textes) où on lit au moins un autre mot (texte) " (Kristeva, 1969, p. 145), ou encore, «tout texte se construit comme une mosaïque de citations, tout texte est absorption et transformation d'un autre texte » (p. 145) ${ }^{3}$. Kristeva attribue l'origine de ce concept à ses lectures de Bakhtine traitant du dialogisme. Certes, chez ce dernier, le mot " intertextualité » ne figure pas, mais ses recherches sur le rôle de l'auteur mènent bien à une vision du texte comme étant lieu d'un croisement de perspectives.

En étudiant divers écrivains, notamment Dostoïevski et Rabelais, Bakhtine relève la nature polyphonique de ces œuvres, voyant la création littéraire comme l'intersection de plusieurs types de discours. Le vieux modèle de l'auteur comme créateur tout-puissant, responsable de tout aspect de son texte, disparaît pour donner lieu à une multiplicité de voix :

[Dans les romans de Dostoïevski], ce n'est pas un grand nombre de destinées et de vies qui se développent au sein d'un monde objectif unique, éclairé par l'unique conscience de l'auteur; c'est précisément une pluralité de consciences, ayant des droits égaux, possédant chacune son monde qui se combinent dans l'unité d'un événement, sans pour autant se confondre. [...] sans devenir le simple objet de la conscience de l'auteur. (Todorov, 1981, p. 33)

Lorsqu'un personnage intervient dans un récit de Dostoïevski, le langage utilisé est donc, selon Bakhtine, unique, porteur de sa

\footnotetext{
3 Pour un bon aperçu et un survol historique de la notion d'intertextualité, voir Intertextualité : Mémoire de la littérature de Tiphaine Samoyault.
} 
propre charge philosophique : chaque personnage « jouit d'une autorité idéologique et d'une parfaite indépendance; il est perçu comme l'auteur de ses propres conceptions idéologiques à valeur absolue, et non pas comme objet de la vision artistique de Dostoïevski » (p. 31). Au départ, l'intertextualité n'était donc pas une notion précise, mais plutôt une approche sur la nature même d'une œuvre, le texte devenant un lieu de rencontre entre différents systèmes de langages. Comme le soulignent Engélibert et Tran-Gervat, «les préoccupations de ses promoteurs étaient d'ordre philosophique - ou «théorique » dans le vocabulaire de l'époque - avant d'être critiques» (p. 8).

Cette notion de dialogue se prête particulièrement bien aux «Doigts extravagants ». L'incipit place la nouvelle à New York: "La quatorzième rue est un chemin qui mène à l'East River » (p.145), mais au fur et à mesure, la ville prend une allure germanique. Les couleurs du drapeau allemand se greffent sur la ville américaine : «À gauche, des couleurs jaunes, rouge brique, brunes, délimitées par des lignes noires qui leur donnaient des formes, des formes de maisons » (p. 145). Un peu plus loin, la narratrice traverse un passage dans lequel est affichée une annonce pour un film allemand: "Mädchen in Uniform» (p.145). C'est une présence perceptible également sur le plan de l'intrigue. La nouvelle met en scène un personnage principal scellant un pacte diabolique, une reprise patente de la légende allemande de Faust, dont l'expression la plus célèbre revient à Goethe. Or, une référence à ce dernier se cache dans le récit. Le nom de l'éditeur du chef-d'œuvre écrit par les doigts extravagants - Steiner - renvoie à Rudolf Steiner (1861-1925), philosophe et créateur du mouvement spirituel de l'anthroposophie fondée sur la version goethéenne 
de Faust 4 . Certes, l'atmosphère germanique régnant dans la ville de New York et les éléments faustiens qui conduisent l'intrigue ne constituent pas un système de langage. Mais ils créent un arrière-plan dont surgira une voix, dans le sens bakhtinien du terme, repérable et idéologiquement indépendante, une voix relevant à notre avis du genre fantastique.

Le fantastique allemand - et peut-être le fantastique du $\mathrm{XIX}^{\mathrm{e}}$ siècle en général - a des racines dans le romantisme. Dans leur ouvrage Visages du double, Jourde et Tortonèse consacrent la plus grande partie d'un chapitre à cette genèse historique. Selon eux, l'explosion de figures doubles (les fantômes, etc.) dans les œuvres, entre autres de Jean-Paul, de Chamisso, d'Hoffman et d'Arnim, coïncide largement avec la montée du romantisme, ayant à sa base l'exploration d'un sujet « dédoublé » :

Depuis Kant [...] la philosophie, et notamment la philosophie allemande est devenue une philosophie du sujet. Le moi se trouve au cœur de la pensée de Fichte, qui domine le premier quart du XIX $\mathrm{X}^{\mathrm{e}}$ siècle. Fichte élabore un système fondé sur une sorte de dédoublement du sujet : le Moi absolu, inconditionné, originaire, engendre le non-moi, le monde extérieur, dans lequel se trouve inclus le moi empirique (p. 27).

Chez Goethe, cette "philosophie du sujet » se manifeste dans la présence presque obsessionnelle de la thématique du fractionnement dans le personnage de Faust - ce dernier se

\footnotetext{
${ }^{4}$ La vie de Steiner est, en fait, principalement consacrée à l'œuvre de Goethe. Il fut l'éditeur scientifique d'une nouvelle édition de Faust en 1882 ainsi que l'éditeur des archives de Goethe à Weimar, écrivit deux œuvres philosophiques sur la pensée de ce dernier, Une théorie de la connaissance chez Goethe et Goethe et sa conception du monde, mit en scène le Faust de Goethe et créa le bâtiment qui existe toujours aujourd'hui, le Goethéaneum. Voir, dans Lachman, le chapitre « À Weimar, aux archives de Goethe ».
} 
trouve dans la sphère physique, mais cherche la totalité à travers le spirituel - ainsi que sur le plan de l'écriture. La pièce est en deux parties, possède deux personnages féminins principaux (Gretchen et Hélène) ainsi que deux forces (Dieu et Méphistolès) ${ }^{5}$.

Cette dialectique romantique imprègne le récit de Maillet et devient non seulement une voix reconnaissable, mais aussi une esthétique sur laquelle repose la nouvelle. Avec l'image des doigts coupés qui deviennent animés, le sujet et, physiquement parlant, le corps, se trouvent dédoublés, fragmentés, répétés :

Lorsque la narratrice retire les doigts du manteau, le lecteur est soudainement confronté à l'image d'un être à trois mains et à quinze doigts. Quelques pages plus loin, c'est un être où main et pied se joignent : la narratrice prend le talon de sa chaussure pour faire "lâcher-prise » aux doigts qui avaient grimpé «le mur de la maison jusqu'à [sa] croisée » (p. 150). Ou encore, un être morcelé lorsque les doigts se trouvent dans un béret, prenant alors la place qu'occupe d'habitude une tête (p.153). (Josephy, p. 60)

En outre, puisque les doigts appartiennent à la main d'un homme, c'est un dédoublement traversé par la problématique du genre. Dans le récit, la répartition par genre est insistante : «Un homme donnait un manteau, comme ça, en pleine rue, à moi, une étrangère, et sans ajouter au geste une seule parole. Pourquoi? » (p. 147), «Il avait donné le manteau à une jeune femme. Pourquoi?» (p. 148), «j'entrai dans ma chambre avec le manteau d'homme» (p.148). L'être mi-femme mi-homme, androgyne, qui en résulte est, encore une fois, ancré dans la philosophie du romantisme allemand6. Insérée dans la

5 Les liens entre l'œuvre de Goethe et le romantisme sont complexes (voir Nicholls).

6 Voir Busst, Monneyron et Friedrichsmeyer. 
structure même de la langue, le lecteur entend chez Maillet une voix qui fait irruption dans le récit au travers d'oppositions typiques du romantisme allemand: moi/autre, sujet/objet, féminin/masculin.

Mais cette voix n'en est qu'une parmi les autres mises en place par le système polyphonique du texte. Par exemple, lorsque la narratrice décrit son appartement, elle relève la présence des «blattes, des rats » et des "cancrelats », après quoi elle ajoute : « (chez nous on dit les coquerelles) » (p. 149). Cet élément contraste avec le récit précédent, non seulement par l'emploi de parenthèses qui crée une séparation visuelle, mais aussi par l'emploi de deux termes pour "cancrelat», le second laissant transparaître un «nous» identitaire et québécois. En fait, le lecteur n'a aucune information biographique sur l'identité nationale/culturelle de la narratrice, ce qui rend le terme «coquerelle» d'autant plus frappant. À l'univers typiquement new-yorkais, aux rues pestilentielles et aux appartements infestés de rats et de «cancrelats » se mêle donc une voix qui exprime sa québécitude ${ }^{7}$.

Les premières définitions de l'intertextualité ont donc l'avantage de montrer, dans le récit, différents systèmes de langage qui, souvent exprimés dans des métaphores, correspondent à des niveaux de discours difficiles à cerner. Où commence et se termine exactement une voix romantique? Comment la repérer et la séparer des autres voix présentes dans le récit? En l'absence d'informations contextuelles, est-il

\footnotetext{
7 Écrite au début de la Révolution tranquille, la nouvelle pourrait être analysée sous un angle historique et politique. Maillet est en effet une écrivaine encore peu étudiée. Dans un des seuls articles qui lui soient consacrés, Biron explore la pluralité linguistique et culturelle des villes, particulièrement celle de Montréal.
} 
possible de trouver les traces d'un discours ancré, comme nous l'indiquent Jourde et Tortonèse, dans la philosophie de Kant ou de Fichte?

Ces questions montrent que le dialogisme est une notion qui peut mener à des analyses plus ou moins subjectives et ouvrir sur des perspectives très larges. En 1987, Marc Eigeldinger, s'inspirant sans doute de Bakhtine, présente ses recherches sur l'intertextualité de la façon suivante :

Mon projet est de ne pas limiter la notion d'intertextualité à la seule littérature, mais de l'étendre aux divers domaines de la culture. Elle peut être liée à l'émergence d'un autre langage à l'intérieur du langage littéraire ; par exemple celui des beauxarts et de la musique, celui de la Bible ou de la mythologie, ainsi que celui de la philosophie (p. 15).

Exprimée ainsi, l'intertextualité semble vaste, trop étendue peut-être, pour être saisissable et risque, comme l'écrit Angenot, " [de] faire du neuf avec du vieux, et par exemple [d']appeler analyse intertextuelle une bien traditionnelle critique philologique des sources et des influences littéraires » (p. 2). En effet, la difficulté de savoir ce qui constitue une voix ou « un système de langage » chez Bakhtine, voire un « texte » chez Kristeva, est au cœur de cette confusion entre ce qui constitue une intertextualité ou une traditionnelle étude des sources ${ }^{8}$.

8 Kristeva était d'ailleurs elle-même consciente de cette difficulté. Dans $L a$ révolution du langage poétique en 1974, elle écrit: «Le terme d'intertextualité désigne cette transposition d'un (ou de plusieurs) systèmes de signes en un autre, mais puisque ce terme a souvent été entendu dans le sens banal de "critique des sources" d'un texte, nous lui préférons celui de transposition, qui a l'avantage de préciser que le passage d'un système signifiant à un autre exige une nouvelle articulation du thétique — de la positionnalité énonciative et dénotative. » (p. 60) 


\section{L'entrée en scène du lecteur}

Après Bahktine-Kristeva, d'autres théoriciens ont essayé d'aller vers plus de spécificité. Dans Palimpsestes (1983), Genette tente de réduire l'étendue de l'intertextualité en créant des catégories et des types d'intertextualité. Lors du colloque Le Plaisir de l'intertextualité, Arrivé décline ainsi les apports de Genette:

on a vu fleurir sur le modèle d'intertextualité, une foule de néologismes. D'abord par mutation du préfixe: Genette a introduit paratextualité, métatextualité', hypertextualité', architextualité. Mais on trouve aussi autotextualité, bibliotextualité, catatextualité, épitextualité, extra- et intratextualité, hétéro- et homotextualité, hypo-textualité, mimotextualité, péritextualité, transtextualité, etc. - chacune de ces formations ayant ses équivalents en -texte et -textuel. Après la mutation du préfixe, sa combinaison avec d'autres : on observe alors intraintertexualité, intermimotextualité, et même — je n'invente rien — "inter-intermimotextualité" (p. 16).

Bien qu'Arrivé ironise sur la capacité infinie du jargon à croître et à se multiplier, il souligne néanmoins l'innovation de Genette. Toute intertextualité n'entre plus dans le même cadre. L'enjeu n'est plus de s'attarder sur l'aspect intertextuel de tout texte, mais de s'informer sur les variations s'opérant dans un récit donné.

La distinction, par exemple, entre intertextualité et hypertextualité montre bien une divergence fondamentale sur le plan du récit. La première vise à découvrir les traces visibles d'autres discours dans un texte. La deuxième s'apparente à la parodie ou au pastiche - lorsqu'un texte présent est dérivé d'un autre, sans nécessairement que les mêmes mots s'y

Ceci ne résout néanmoins pas la difficulté qui consiste à distinguer « texte » et « source », puisqu'une source a vocation à être transposée. 
retrouvent ${ }^{9}$. Dans le cas de la nouvelle de Maillet, les deux catégories sont présentes. Des fragments de phrases empruntés à d'autres écrivains apparaissent dans la nouvelle et sont donc un exemple classique d'intertextualité, comme la conceptualise Genette. Quant à l'hypertextualité, les doigts coupés qui s'animent constituent une image dérivée de la Bible. Dans le livre de Daniel, cinq doigts coupés commencent à écrire sur le palais d'un mur babylonien. À la rigueur, l'exemple des doigts détachés pourrait aussi être classé dans la catégorie de l'architextualité puisque c'est une image présente dans de nombreuses œuvres fantastiques. Le pastiche ou la parodie visent en l'occurrence non pas une œuvre spécifique, à savoir, la Bible, mais tout un genre fantastique, ou, plus particulièrement, dans le cas de la thématique du pacte diabolique, la souscatégorie de textes ayant pour motif un pacte faustien.

La méthodologie de Genette présente donc le grand avantage de montrer à quel point la notion d'intertextualité est étendue et peut être mieux appréhendée par différentes taxinomies. Cependant, des questions demeurent sur le plan herméneutique, et plus particulièrement sur les implications du rôle du lecteur. Comment celui-ci détermine-t-il si un élément est architextuel, intertextuel, hypertextuel, etc., et à quoi bon? Ce sont des questions qui suscitent une réflexion sur la nature dialectique de l'intertextualité - le texte comme point de départ et le lecteur comme point d'arrivée - , aspect que Barthes et Riffaterre sont peut-être les premiers à avoir mis au jour. Une dizaine d'années avant l'ouvrage de Genette, Barthes

9 « J'appelle donc hypertexte tout texte dérivé d'un texte antérieur par transformation simple (nous dirons désormais transformation tout court ou par transformation indirecte : nous dirons imitation. » (Genette, 1983, p. 14). 
débute son exploration axée sur le lecteur. En 1973, dans Le Plaisir du texte, il écrit :

Je savoure la règle des formules, le renversement des origines, la désinvolture qui fait venir le texte antérieur du texte ultérieur. Je comprends que l'œuvre de Proust est, du moins pour moi, l'œuvre de référence, la mathésis générale, le mandala de toute la cosmogonie littéraire [...]. Proust, [...] ce n'est pas une " autorité », simplement un souvenir circulaire. Et c'est bien cela l'inter-texte: l'impossibilité de vivre hors du texte infini - que ce texte soit Proust, ou le journal quotidien, ou l'écran télévisuel: le livre fait le sens, le sens fait la vie. (p. 59)

Cette définition de l'intertextualité se rapproche beaucoup de celle de Kristeva, à savoir que tout texte est «absorption et transformation d'un autre texte », « une mosaïque de citations » (Kristeva, 1969, p. 145) ${ }^{10}$. Cependant, Barthes se démarque de ses prédécesseurs par l'importance qu'il donne au rôle du lecteur, ce «je» qui comprend la «règle des formules» de l'écriture et qui peut reconnaître dans un texte présent un texte antérieur. C'est sur ce point également que l'on voit la rupture avec les travaux à venir de Genette, car l'accent est mis non pas sur la volonté de classer et de nommer des phénomènes, mais sur le rapport qui existe entre texte et lecteur.

Riffaterre explore davantage ce rapport et se concentre de façon minutieuse sur les « règles » du langage auxquelles fait allusion Barthes. Avec la publication de La Production du texte en 1979, l'intertextualité se voit reliée à une façon de comprendre la structure d'un texte et, donc, de le lire. Selon Riffaterre, tout texte a une logique propre et, en l'absence de celle-ci, le lecteur doit chercher une logique ailleurs, dans un

10 Orr (p. 32-36) mène une défense ardente de Kristeva, montrant l'originalité de celle-ci par rapport à Barthes. 
intertexte. L'exemple qu'il tire d'un texte de Baudelaire rend son positionnement intertextuel particulièrement compréhensible: «Il y avait des vieilles mères portant des avortons accrochés à leurs mamelles exténuées » (p. 56). Selon Riffaterre, il n'y a pas de "chaîne associative » (métonymique, métaphorique ou autre) permettant de dériver "accroché du mot avorton» (p. 56). Autrement dit, la dissonance ou « agrammaticalité » des mots à l'intérieur d'un vers nécessite le recours à un intertexte. Dans ce cas, ce sera la phrase plutôt stéréotypée des mères portant des nourrissons suspendus à leurs seins (p.57). Le vers de Baudelaire se construit alors en opposition à une phrase déjà existante : le mot "mères ", qui dans son sens positif se relie à la jeunesse, devient, «vieilles mères », le mot "nourrissons » se transforme en « avortons » et le verbe «suspendre» est remplacé par «accrocher», verbe qui, selon Riffaterre, comporte des connotations négatives. À cet égard, «le texte guide le lecteur vers une interprétation correcte» (p. 155). C'est dans sa production même que le lecteur ressent instinctivement un intertexte, poussé par une curiosité et un désir de trouver une logique que, parfois, seul un recours à l'extérieur peut combler.

\section{Riffaterre mis en pratique}

L'intérêt de cette nouvelle forme d'intertextualité devient évident à la lumière de la nouvelle de Maillet. Le récit contient plusieurs événements qui provoquent des pauses lors de la lecture, dont l'un des plus marquants se produit lorsque l'étranger donne à la narratrice le manteau contenant les doigts coupés. Ce personnage est décrit comme marchant « rapidement » et «son bras gauche levé en équerre devant lui 
se terminait par un gant de boxe » (p. 146). L'image est étrange et suscite une série de questions: pourquoi «rapidement»? Pourquoi « en équerre »? Pourquoi le bras "gauche » et non le droit? Et, surtout, quel intérêt à faire apparaître un "gant de boxe »? Avant l'arrivée de l'étranger, il n'y a rien dans le récit qui puisse se relier métonymiquement, métaphoriquement ou d'une autre façon à un gant de boxe; le champ lexical des mathématiques évoqué par le mot «équerre» se manifeste dans la phrase suivante : " quand nous fûmes parallèles », mais ces deux exemples font partie d'une seule description et n'ont aucune occurrence ou correspondance ailleurs dans le récit. Il n'y a pas non plus de sons ou de métaphores à l'intérieur de la phrase pour expliquer la description de l'homme qui marche avec un gant de boxe. Selon Riffaterre, c'est une dissonance qui suscitera donc une recherche à l'extérieur du texte.

Recherche qui mène justement à la découverte d'une autre intertextualité : un film des années 1940, de Michel Tourneur, La Main du diable, dans lequel un artiste entreprend un pacte diabolique afin d'être reconnu pour ses peintures. Une fois le pacte scellé, il perd immédiatement sa main gauche. Le recours à un film pose, bien sûr, un problème à l'égard d'une étude sur l'intertextualité. Nulle part dans La Production $d u$ texte, Riffaterre n'aborde la possibilité d'un intertexte filmique, d'autant plus que son argumentation repose sur les chaînes associatives entre signifiants et, donc, sur une analyse fondée sur des bases linguistiques. Depuis, cette zone d'ombre entre texte et image a donné lieu à un nouveau concept, celui d'« intermédialité » (voir Guillemette et al.). En dépit de la différence des langages employés, le film contient bien l'image curieuse de l'homme tenant un manteau avec un bras levé en équerre. En l'absence d'effets spéciaux, l'acteur doit feindre une 
amputation en rassemblant sa main dans un poing, la couvrant d'un gant noir et en levant son bras en angle droit. La main disparaît ainsi dans l'arrière-plan du champ noir. Plus spécifiquement, la phrase de Maillet se construit en relation avec la première scène du film. Pendant les toutes premières minutes, le peintre entre dans un café, son manteau drapé sur l'avant-bras, son bras levé en équerre. Il s'assoit et raconte son histoire au public. Chez Maillet, le gant noir devient gant de boxe en relation avec l'image d'un coup de poing, l'amputation de la main gauche de l'homme devient les doigts d'une main gauche et le manteau drapé de façon nonchalante sur l'avantbras du peintre devient l'endroit où la narratrice découvre les maudits doigts extravagants ${ }^{11}$.

En outre, ces premières phrases de la nouvelle de Maillet ouvrent la porte à d'autres agrammaticalités. Les doigts trouvés dans le manteau sont décrits comme "coupés au-dessus des phalanges et reliés entre eux par un lacet» (p. 150). C'est une image et une construction langagière qui provoquent encore une hésitation lors de la lecture. Il y a bien une allitération avec la lettre «l», mais dans le langage courant, c'est la main qui écrit et non les doigts. Pourquoi donc, dans un texte où une main écrit à la place d'une autre, est-il question de doigts coupés? C'est une incongruité qui pourrait peut-être trouver sa raison d'être dans sa coïncidence avec la Bible, où il s'agit spécifiquement de doigts scripteurs: «En ce moment,

\footnotetext{
${ }^{11}$ D'ailleurs, si ces liens entre les deux œuvres ne suffisent pas, le film renvoie aux autres intertextualités par sa proximité avec l'Allemagne. Réalisé pendant la Seconde Guerre mondiale lorsque la domination de l'Allemagne était à son plus fort, le film est produit par la compagnie Continental, financée en grande partie par des capitaux allemands lors de l'Occupation.
} 
apparurent les doigts d'une main d'homme, et ils écrivirent, en face du chandelier, sur la chaux de la muraille du palais royal. Le roi vit cette extrémité de main qui écrivait. » (Dan., 5:5). Les liens sont évidents : non seulement il s'agit de doigts coupés appartenant à une main masculine, mais l'accent est aussi mis sur les bouts des doigts: les "extrémités » dans le cas de la Bible, les doigts « coupés au-dessus des phalanges » dans le cas de la nouvelle12.

Néanmoins, cette image curieuse de doigts coupés pourrait tout aussi facilement trouver une correspondance avec une autre œuvre de l'intertexte, encore une fois, celle de Tourneur. Dans le film, quand l'artiste scelle le pacte diabolique, il reçoit une boîte dans laquelle se trouve une main gauche d'homme. La main reste immobile, fixe, cachée dans la boîte, élément qui a provoqué une controverse lors du tournage. Contre l'avis du cinéaste, Jean-Paul Le Chanois, son assistant, Jean Devaivre, propose de capter une image des doigts bougeant dans la boîte. Cette anecdote, documentée dans le film Laissez-passer de Bertrand Tavernier sur les films français tournés lors de l'Occupation, aboutit à un compromis. Le Chanois accepte de capter un seul doigt bougeant momentanément, presque inaperçu au moment où le peintre ouvre la boîte dans les dernières minutes du film. L'image des doigts coupés dans la nouvelle de Maillet pourrait donc constituer un clin d'œil à cette scène. D'ailleurs, c'est un rapport qui ressort davantage en prenant en compte le titre de la

\footnotetext{
${ }^{12}$ Le fait que les doigts écrivant appartiennent à une main masculine constitue une critique du machisme de l'institution littéraire. En outre, l'image des «bouts des doigts » a des sous-entendus clairement sexuels (voir Josephy, p. 59-67).
} 
nouvelle. L'adjectif « extravagants » contient un jeu de mots. Les doigts extravagants apportent bien sûr des gains " extravagants", mais étymologiquement, l'épithète fait allusion à un mouvement : extra, venant du latin et voulant dire "au-delà de », "à l'extérieur de», et vagant, venant de l'allemand et voulant dire " errant». Le second sens du titre se résume alors aux doigts qui bougent au-delà de leur limite, tout comme le doigt bougeant dans le film.

Les possibilités intertextuelles ne s'arrêtent cependant pas là. Le choix de se concentrer sur les doigts pourrait également renvoyer à une nouvelle de Gérard de Nerval, «La main enchantée ». Ce texte, paru d'abord en 1832 sous le titre «La main de gloire. Histoire macaronique », raconte l'histoire d'un faible jeune homme qui fait un pacte pour gagner un duel contre un adversaire plus fort que lui. Sa main s'imprégnant d'un pouvoir surnaturel, il tue son rival (quoiqu'il n'ait jamais voulu se battre à mort), éprouve dès lors une angoisse terrible et est condamné à mort. Après son exécution, sa main continue à vivre, "possédée ». L'avant-dernier paragraphe met l'accent sur ces phalanges vagabondes :

[La main] fit un bond prodigieux et tomba sanglante au milieu de la foule, qui se divisa avec frayeur; alors, faisant encore plusieurs bonds par l'élasticité de ses doigts, et comme chacun lui ouvrait un large passage, elle se trouva bientôt au pied de la tourelle du Château-Gaillard ; puis, s'accrochant encore par ses doigts, comme un crabe, aux aspérités et aux fentes de la muraille, elle monta ainsi jusqu'à l'embrasure où le bohémien l'attendait. (p. 56)

Le « crabe », animal bien-aimé de Nerval, est peut-être la source de l'image de doigts « coupés au-dessus des phalanges et reliés entre eux ». Hypothèse d'autant plus plausible qu'on imagine 
que les doigts, dans la nouvelle de Maillet, sont "sanglants", puisque coupés, et qu'ils manifestent un comportement semblable à ceux de Nerval, notamment dans leur désir de grimper aux murailles.

Que faire alors de toutes ces inférences intertextuelles? La phrase concernant les doigts coupés renvoie-t-elle à la Bible, au film, au texte de Nerval? Cette ambiguïté montre à la fois l'avantage et la difficulté des innovations proposées par Riffaterre sur l'intertextualité. Lorsqu'il y a une relation moins évidente (mais néanmoins existante) entre deux œuvres, quelle proximité est nécessaire pour que le lecteur puisse affirmer ou non qu'il s'agit d'une intertextualité? Et si une phrase, comme c'est le cas ici, peut être reliée à plusieurs phénomènes intertextuels - une situation qui arrive sans doute souvent étant donné que les intertextualités se produisent souvent en chaîne, une référence en entraînant une autre - , comment trouver soit l'origine, soit le texte visé par l'auteur? Enfin, cet exemple soulève également le fait que le lecteur peut se tromper, une éventualité tout aussi envisageable ici. Les cinq doigts coupés sont reliés entre eux par un lacet de bottine. L'image du « lacet » ne se trouve nulle part dans les intertextes proposés. Le seul point de rapport pourrait être la main sanglante qui s'agite " au pied » de la tourelle dans la nouvelle de Nerval. Le pied du mur pourrait, dans un glissement intertextuel, évoquer un pied réel, menant donc au «lacet de bottine» dans "Les doigts extravagants ». Mais est-ce assez pour confirmer le texte de Nerval comme étant l'intertextualité voulue par Maillet ou faudrait-il trouvé une autre intertextualité? Pour Riffaterre, répondre à ce genre de questions n'est pas l'essentiel. Ni la culture ni la bibliothèque du lecteur ne le préoccupent. L'important est que le lecteur puisse 
lire « circulairement » en revisitant les particularités d'un texte et reconnaître, de ce fait, l'existence ou non d'un intertexte.

\section{Une « anthropologie de l'altérité »}

Cette préoccupation de la production du texte conduit à un nouveau questionnement. Qu'il y ait une logique propre à chaque texte, n'est-ce pas aussi un type d'intertextualité? Autrement dit, lorsque chaque signifié en déclenche un autre, prescrivant, selon le lexique riffaterrien, son propre mot ou sa propre production, n'y a-t-il pas dans ce mode presque préétabli du langage l'écho d'autres discours ${ }^{13}$ ? Quand Flaubert, par exemple, se met à sa table d'écriture, cherchant minutieusement le mot, la lettre, voire le son juste, se donnant, comme le suggère Zola «un mal infini pour éviter les consonances fâcheuses, les redoublements de syllabes » (n. 24, p. 40), n'est-ce pas là la volonté de créer une œuvre qui répond à une logique du langage, logique prédéterminée par les discours écrits précédemment?

En effet, déjà dans l'œuvre de Bakhtine, il y a l'idée que tout langage est une contamination. Todorov l'a relevé à juste titre :

L'orientation dialogique est, bien entendu, un phénomène caractéristique de tout discours. C'est la visée naturelle de tous les discours vivants... Seul l'Adam mythique, abordant avec le premier discours un monde vierge et encore non dit, le solitaire Adam, pouvait vraiment éviter absolument cette réorientation

\footnotetext{
13 À noter que, pour Riffaterre, l'absence de dissonance est la marque de « l'unicité » de l'œuvre, du style de l'écrivain. Or, l'absence de dissonance peut aussi être considérée comme la marque d'une norme, comme un témoignage du déjà-écrit.
} 
mutuelle par rapport au discours d'autrui, qui se produit sur le chemin de l'objet. (p. 98)

Chaque texte se fonde alors sur un texte préexistant. La collocation même devient la marque d'un discours déjà dit. Pour Laurent Jenny, cette prise en compte de l'Autre est l'élément le plus saillant dans la pensée de Bakhtine: "Son dialogisme implique une certaine conception de l'homme que l'on pourrait définir comme une anthropologie de l'altérité, c'est-à-dire que l'autre $\mathrm{y}$ joue un rôle essentiel dans la constitution du moi. » Pour Bakhtine, cet Autre n'est pas un adversaire. Les expressions comme «polyphonie», « dialogisme » et «pluralité » ont une connotation positive, le texte étant lieu de partage.

À la lumière de la production d'un texte, il faut cependant se poser la question de savoir pourquoi certains discours sont plus influents que d'autres. Pourquoi certaines œuvres pèsentelles plus que d'autres? Maillet est tout à fait consciente de cette domination et la textualise. Lorsque les doigts coupés terminent leur œuvre, le président jette «à peine les yeux sur le tas de feuilles » et offre à la narratrice " un contrat magnifique » et " une avance de dix mille dollars sur [s]es royautés à venir » (p. 154). Quand les doigts écrivent un second texte, la narratrice devient « plus connue qu'Einstein, plus célèbre qu'une étoile de cinéma» (p. 154). L'emploi de ces hyperboles souligne à quel point le poids d'une œuvre, son succès et donc sa place dans la culture commune se fondent sur les choix normatifs des groupes dominants.

L'analyse des «Doigts extravagants » de Maillet permet ainsi de mesurer les apports tout comme les limites des différentes théories de l'intertextualité et la complémentarité 
de ces approches. Mais la nouvelle apporte aux théories fondatrices de l'intertextualité une dimension supplémentaire, celle de la subjectivité de la fiction. Là où les théoriciens de la littérature ne font qu'envisager un jeu intellectuel, l'auteure pointe l'impact et les séquelles de la dimension intertextuelle sur le processus créatif. En effet, au fil du texte, les doigts deviennent de plus en plus puissants, au point que le récit se termine sur la possibilité effrayante de doigts meurtriers : «Or, [les doigts] m'aiment et devinent sans doute ma plus secrète pensée. N'ont-ils pas ce matin encerclé ma gorge avec plus de vigueur? » (p. 156). Cette métaphore évidente de l'incapacité de sortir de l'emprise des mains/mots des autres devient également le lieu d'une contamination. L'image des doigts qui étranglent ne relance-t-elle pas un autre chaînon intertextuel à partir de la phrase célèbre de «La main d'écorchée » de Maupassant, où après avoir reçu une main coupée, le narrateur se réveille et porte «au cou les marques de cinq doigts qui s'étaient profondément enfoncés dans la chair » (p. 5)?

\section{Bibliographie}

Angenot, Marc. (1983), «L'intertextualité : enquête sur l'émergence d'un champ notionnel », Revue des sciences humaines, vol. 189, nㅡㄴ 1, p. 121-135.

BaKhtine, Mikhaïl. (1970 [1929]), La Poétique de Dostoïevski, Paris, Seuil. 
Barthes, Roland. (1973), Le Plaisir du texte, Paris, Seuil.

Biron, Michel. (1996), «Profil de Montréal chez Andrée Maillet », La nouvelle au Québec, t. IX, Ottawa, Fides.

Bloom, Harold. (1973), The Anxiety of Influence, New York, Oxford University Press.

Busst, A. J. (1967), "The Image of the Androgyne in the Nineteenth Century", in Ian Fletcher (ed.), Romantic Mythologies, London, Routledge and Kegan Paul, p. 1-95.

Compagnon, Antoine. (1979), La Seconde Main ou le travail de la citation, Paris, Seuil.

Clayton, Jay et Eric Rothstein. (1991), Influence and Intertextuality in Literary History, Madison, Madison Wisconsin Press.

EIGELDINGER, Marc. (1987), Mythologie et intertextualité, Genève, Slatkine.

EnGÉLIBERT, Jean-Paul et Yen-Maï Tran-Gervat. (2008), « Avantpropos», dans Jean-Pierre Eigeldinger et Yen-Maï TranGervat (dir.), La Littérature dépliée: reprise, répétition, réécriture. Rennes, Presses universitaires de Rennes, p. 7-10.

FriedrichSMEYER, Sara. (1983), The Androgyne in Early German Romanticism, Bern, Peter Lang.

GENETTE, Gérard. (1982), Palimpsestes : La littérature au second degré, Paris, Seuil.

Goethe, Wolfgang van. (1969 [1832]), Faust et le second Faust, trad. Gérard de Nerval, Paris, Garnier. 
GUILLEMETTE, Lucie, Louis HÉBERT et al. (2009), Intertextualité, interdiscursivité et intermédialité, Québec, Les Presses de l'Université Laval.

JEnNY, Laurent. (2003), «Dialogisme et Polyphonie », Méthodes et problèmes, Genève, Dpt de français moderne, http://www.unige.ch/lettres/framo/enseignements/metho des/dialogisme/.

Jourde, Pierre et Paolo ToRTONESE. (1996), Visages du double : Un thème littéraire, Paris, Nathan.

JoSEPHY, Rebecca. (2010), « Mains (in)visibles : Intertextualité, intratextualité et autoréférentialité dans "Les doigts extravagants" d'Andrée Maillet », Voix plurielles, vol 7, no 1, p. $52-78$.

KRISTEVA, Julia. (1969), Seméiotikè, recherches pour une sémanalyse, Paris, Seuil.

—. (1974), La Révolution du langage poétique, Paris, Seuil.

LACHMAN, Gary. (2009), Rudolf Steiner, trad. Christine Piot, Paris, Actes Sud.

MAILlET, Andrée. (1987 [1963]), "Les doigts extravagants.», dans Anthologie de la nouvelle et du conte fantastiques québécois, Louiseville, Fides, p. 145-156.

MAUPASSANT, Guy de. (1974 [1875]), « La main d'écorché », dans Contes et nouvelles, Paris, Gallimard, p. 3-8.

MONNEYRON, Frédéric. (1994), L'Androgyne romantique: du mythe au mythe littéraire, Grenoble, Édition littéraire et linguistique de l’Université de Grenoble. 
NERVAL, Gérard de. (1912 [1832]), Poésies, La main enchantée, Sylvie, Voyage en Orient, Londres, J.M. Dent \& Sons.

NiCHOLLS, Angus. (2002), « Goethe, Romanticism, and the AngloAmerican Critical Tradition», Romanticism on the Net, vol. 28, novembre, n.p.

ORR, Mary. (2003), Intertextuality: Debates and Contexts, Cambridge, Polity Press.

Riffaterre, Michaël. (1979), La Production du texte, Paris, Seuil.

SAMOYAULT, Tiphaine. (2001), L'Intertextualité : mémoire de la littérature, Paris, Nathan.

STEINER, Rudolph. (1928), Goethe's Conception of the World, New York, Anthroposophic Press.

-. (1925), Goethe's Standard of the Soul, London, Anthroposophical Publishing Company.

THEIS, Raimund, Hans T. SIEPE et al. (1986), Le plaisir de l'intertexte: formes et fonctions de l'intertextualité : roman populaire, surréalisme, André Gide, Nouveau Roman. Actes du colloque à l'Université de Duisburg, vol. 3, Frankfurt am Main, P. Lang.

Todorov, Tzvetan. (1981), Mikhaïl Bakhtine: Le principe dialogique, Paris, Seuil.

—. (1979), «Bakhtine et l'altérité », Poétique, vol.40, p. 502513.

Tourneur, Maurice. (1943), La Main du diable, scénario de JeanPaul Le Chanois, 82 min.

ZolA, Émile. (1957 [1877]), Amis de Flaubert, Rouen, Association des Amis de Flaubert, no 24, p. 40. 


\title{
Résumé
}

Dans la nouvelle fantastique "Les doigts extravagants » d'Andrée Maillet, une artiste découvre cinq doigts dans la poche d'un manteau. Les doigts s'animent, la tourmentent et commencent à écrire un chef-d'œuvre sur sa table de cuisine. Thématiques évidentes de ce récit, l'anxiété de l'écriture et le poids du passé s'inscrivent également dans la structure du texte, le lecteur ressentant la présence d'autres œuvres fantastiques. Que peut apporter cette nouvelle hantée par l'origine des mots au débat sur l'influence et l'intertextualité? À la lumière de décennies de travaux dans le domaine, cet article explore comment la théorie éclaire, informe ou échoue face à un même texte.

\begin{abstract}
In the fantastical short story "Les doigts extravagants" by Andrée Maillet, an artist finds five fingers in the pocket of coat. The digits come alive, torment her, and proceed to write a masterpiece on her kitchen table. The anxiety of writing in the wake of past great works - an obvious theme in this story - also permeates the narrative, as the reader senses an almost constant presence of other fantastic texts. What can this short story haunted by the origin of words bring to the debate on Influence and Intertextuality? In light of decades of criticism on these subjects, this article explores how theory informs, illuminates and sometimes comes up short against one text.
\end{abstract}

